\title{
Zu Depression und transgenerational weitergegebenem Trauma: ein psychoanalytischer Kommentar
}

\author{
Mariia Lenherr
}

Online publiziert: 3. Dezember 2019

(C) Der/die Autor(en) 2019

Zusammenfassung Dieses Paper behandelt die mögliche Interrelation von Depression mit generationsübergreifend weitergegebenen Traumata aus der Sicht der Psychoanalyse. Die Autorin unternimmt den Versuch, Trauern und Melancholie voneinander abzugrenzen soweit es das aktuelle Verständnis von Depression betrifft. Das Phänomen der verschiedenen depressiven States auf einem individuellem Level wird untersucht, so wie Freud und nachfolgende PsychoanalytikerInnen es vorgeschlagen haben, einschließlich produktiver und unproduktiver Depression (Gut). Zum Verständnis generationsübergreifend weitergegebener Traumata wird eine kurze Analyse der diesbezüglichen Schriften, besonders den Holocaust betreffend, vorgestellt. Die Autorin bespricht die Auswirkungen und Mechanismen von generationenübergreifend weitergegebenen Traumata, wie sie in den einflussreichen Schriften von Laub, Salberg, Bromberg, Faimberg und anderen behandelt werden. In Bezug auf Gruppenlevel wird Depression als Konsequenz von Verlusten und Traumatisierung besprochen denen jeder von uns als Teil der Gesellschaft ausgesetzt ist. Das Paper endet mit Schlussfolgerungen innerhalb derer Anwendungen in der Psychoanalyse thematisiert werden.

Schlüsselwörter Depression - Depressive Position · Psychoanalyse · Generationsübergreifend weitergegebenes Trauma · Historisches Trauma · Transgenerational weitergegebene Traumata

\section{Depression and transgenerationally transmitted trauma: a psychoanalytic comment}

Summary This paper discusses the possible interrelation of depression and transgenerationally transmitted trauma from the perspective of psychoanalysis. The author attempts to demarcate mourning and melancholia in respect to the contemporary understanding of depression. The phenomenon of depressive states on the individual level is inspected as suggested by Freud and later psychoanalysts, including productive and unproductive depression (Gut). To understand transgenerationally transmitted trauma the short analysis of psychoanalytic writings on historical trauma, especially the Holocaust, is presented. The author discusses the effects and mechanisms of transgenerationally transmitted trauma, as found in influential writings of Laub, Salberg, Bromberg, Faimberg, and others. On the group level, depression is discussed as a consequence of losses and traumatisation that each of us as a member of society is exposed to. The paper is wrapped up with conclusive remarks, where applications of psychoanalysis are shortly discussed.

Keywords Depression - Depressive position · Psychoanalysis - Transgenerationally transmitted trauma $\cdot$ Historical trauma

Was du ererbt von Deinen Vätern hast, erwirb es, um es zu besitzen. Was man nicht nützt, ist eine schwere Last (Faust I, Goethe 1949).

\section{Einleitung}

Depression ist weltweit zu einem der großen Gesundheitsprobleme geworden. Wie kann es sein, dass in der westlichen Welt, mit all ihren fachlichen und me- 
dizinischen Errungenschaften, immer noch eine derartige Hilflosigkeit herrscht, wenn es um dieses Rätsel der Psyche, die Depression, geht?

Bevor ich mich dem zuwende, möchte ich Sie als Leserin oder Leser einladen, mit mir die Frage zu betrachten, was als Gesellschaft unser Beitrag zu dieser Epidemie Depression ist, mit der wir in industrialisierten Hochkulturen heute konfrontiert sind. In diesem Paper wird das Thema durch die Brille der Psychoanalyse angeschaut werden, und diese hat eine lange Forschungstradition im Bereich Depression. Dabei verwende ich sowohl die klinische und theoretische Sicht der Psychopathologie als auch kritische Sozialtheorie.

Frau T. sucht psychotherapeutische Hilfe wegen ihrer wiederkehrenden depressiven Symptome: Selbstzweifel, sexuelle Empfindungslosigkeit, Erschöpfungssyndrom, Apathie, Gefühle von Schuld und Inadäquatheit, Selbstentwertung, Affekthemmung, Hemmung der Denkprozesse, Mattheit und schließlich Schlafstörungen, bei denen eine bestimmte Art von Albtraum ihre Träume dominiert. Sie berichtet oft davon, vom Krieg zu träumen, davon, in Gefahr zu sein, Soldaten zu sehen, Panzer, von der Dringlichkeit zu fliehen und sich zu verstecken. Frau T. ist in ihren 20ern, also warum würde ein Mensch, der beinah 50 Jahre nach dem Ende des 2. Weltkrieges geboren worden ist, solche Träume haben? Sie verfügt über kein besonderes Wissen zur Geschichte ihrer Familie während des Krieges und weiß nur, dass ihr Großvater auf Seiten der Sowjetunion als Soldat gekämpft hat.

Dies ist nur ein Beispielfall, in dem wir, um die depressiven Symptome der Patientin zu verstehen, über die Fragen nach einer symbiotischen Dyade mit der Mutter, einer ödipalen Situation mit dem Vater oder einer möglichen lateralen Konkurrenz zu einem Bruder oder einer Schwester hinausgehen müssen. Wir müssen alles mit dem Fernrohr über die Generationen hinweg betrachten („to telescope of generations“) wie Faimberg (2005) es so treffend formuliert. Und hierbei sollten wir gezielt auf das Thema Trauma fokussieren. Der beschriebene Fall hat dazu geführt, dass ich angefangen habe, Depression als soziales Phänomen in Augenschein zu nehmen, sowie ihre Verbindung mit generationsübergreifend weitergegebenem Trauma zu betrachten.

Die Geschichte der Menschheit könnte als Geschichte einander traumatisierender Gruppierungen neu geschrieben werden. Neben den beiden Weltkriegen überschatten unsere Geschichte auch militärische, ethnische und religiöse Konflikte und Grausamkeiten von mehr lokalem Charakter - wobei sich in Zeiten der Globalisierung die Frage stellt, was „lokal“ heute bedeutet. Um nur einige $\mathrm{zu}$ nennen: der Genozid an den Armeniern, die Pogrome in Osteuropa, der Holodomor (das planmäßige Töten durch Entzug sämtlicher Nahrungsreserven der Bevölkerung unter Stalin in den Jahren 1932-1933) in der Ukraine, die ein Jahrtausend währenden Periode des araboisla- mischen Sklavenhandels in Afrika, die Gräuel von Srebrenica, und viele andere. Was bedeutet das, wenn wir uns dem Thema Depression zuwenden?

Depression ist eine der weitverbreitetsten psychischen Erkrankungen. Weltweit leiden nach aktuellen Schätzungen rund 322 Mio. Menschen, das sind 4,4\% der Bevölkerung, an Depressionserkrankungen. 8\% der österreichischen Bevölkerung (rund 550.000 Personen) haben eine ärztlich diagnostizierte Depression oder geben von selbst an, depressiv $\mathrm{zu}$ sein. Schätzungen zufolge verursachen Depressionen mit 92 Mio. $€$ rund ein Drittel aller Gesamtkosten psychischer Erkrankungen. Depressionserkrankungen sind damit die teuerste psychische und neurologische Erkrankung in Europa (Bundesministerium für Arbeit, Soziales, GesundheitundKonsumentenschutz 2019, S. 33). Die angegebene Statistik schreit geradezu danach, sich dem Thema Erkrankungen aus dem depressiven Spektrum und ihrer Erforschung und Behandlung neu zu widmen.

Die Hauptfrage, die ich in diesem Paper behandeln möchte, ist diese: Spielen generationsübergreifend weitergegebene Traumata in Bezug auf depressive Verfassungen eine Rolle, und falls dem so ist, was sind dann die kollektiven Auswirkungen davon? In diesem Paper bedeutet „Großgruppe“ jeweils eine größere Anzahl von Personen, die über eine gemeinsame Identität verfügen (geographisch, geschichtlich, dem gleichen historischen Trauma ausgesetzt gewesen).

Warum ist es für das Feld der Psychoanalyse von Bedeutung, sich damit auseinanderzusetzen? Warum ausgerechnet jetzt? Der Zustand der „Massenamnesie“ in dem wir, als Gesellschaft, uns befinden, neigt zur schmerzlichen Wiederholung vergangener Traumata, und auch zum Leugnen historischer Fakten, solange, bis Anerkennung, Zeugnis-Ablegen und Trauer erfolgt sind. Das letztendliche Ziel der Psychoanalyse ist die Suche nach der Wahrheit, das, was Bion den Drang zur Wahrheit, den „truth drive“, nennt (Grotstein 2007).

Maria Yellow Horse Brave Heart definiert geschichtliches Trauma als „kumulative emotionale und psychologische Verletzung über die Lebensspanne einer Generation und darüber hinaus, ausgehend von einem starken Gruppentrauma“. Aktuelle Forschungsergebnisse der Neurowissenschaft verändern das Paradigma innerhalb der Psychoanalyse und gestatten uns einen flüchtigen Blick in die Auswirkungen von Trauma auf unsere Gene.

Trotzdem sich dieser Text natürlich, wie schon an seinem Titel erkennbar, mit psychoanalytischen Theorien auseinandersetzt, muss doch auch erwähnt werden, dass sich andere therapeutische Schulen ebenso mit dem Thema beschäftigt haben. Insbesondere systemische Zugänge haben das Gebiet der transgenerational weitergegebenen Traumata betrachtet, wie zum Beispiel Lempa (2000), ein systemischer Therapeut, der postuliert hat, Trauma habe einen „infek- 
tiösen Charakter, es wirkt wie ein ,chronisches Gift (Lempa 2000), innerhalb von familiären Beziehungen und über Generationen hinweg“. Auch Korittko und Pleyer (2010), Trepper und Barrett (1991) haben sich mit dem Nachhall traumatischen Geschehens in Familien befasst.

Sprachverwirrungen oder das Wortschatz-Problem: das psychoanalytische Verständnis von Depression und generationsübergreifend übertragenem Trauma

Man könnte meinen, dass Depression heute die häufigste falsch verwendete Diagnose der Psychologie und Psychiatrie darstellt. Als praktizierende Therapeutin, die täglich mit PatientInnen arbeitet, begegne ich ständig Menschen, die mit „Depression“ diagnostiziert worden sind. Der Begriff ist so weitverbreitet, verwendet und missbraucht, dass er ganz alltäglich wirkt. Er ist Teil unseres Alltagswortschatzes, beinahe ein Routinewort, etwas Normales. Gibt es so etwas wie eine „normale Depression“?

Das Konzept von Klein (1940) namens „depressive Position“ bezieht sich auf eine angenommene Phase komplexer intrapsychischer Prozesse, die sich ihrer Meinung nach in einem normalen Kind bis zum Alter von 5 Monaten entwickelt haben und dann im Laufe der weiteren Entwicklung überwunden werden müssen.

Bei der depressiven Position besteht eine Beziehung zu einem integrierten Elternbild, das sowohl geliebt als auch gehasst wird. Das Individuum erkennt seine destruktiven Wünsche gegenüber dem Objekt. Das bringt eine gewisse charakteristische Traurigkeit mit sich, doch dem entspricht auch ein stärker integriertes Ich (Fonagy 2001). Es scheint demzufolge, dass eine bestimmte Traurigkeit, die umgangssprachlich auch als Depression bezeichnet wird, Teil der normalen psychischen Entwicklung ist.

Freud verwendet in seinen Schriften den Begriff der „Melancholie“. Bereits im Jahr 1917 unterscheidet er zwischen Melancholie und Trauer, wobei letztere die Reaktion auf den Verlust eines Objektes außerhalb des Patienten und dem Bewusstsein zugänglich ist. Trauer bedarf keiner Behandlung, da sie keine Pathologie darstellt. Eine weitere wichtige Unterscheidung liegt darin, dass es bei Trauer keine Störung der Selbstachtung gibt (Freud 1917, S. 244). Sobald die Trauerarbeit abgeschlossen ist, ist das Ego wieder frei und unbefangen („ego is free and uninhibited again“ (ibid., S. 245)). Bei Trauer wird die Welt leer und armselig, bei Melancholie hingegen das Ego (ibid., S. 246). Die Selbstvorwürfe und der Wunsch nach Selbstbestrafung bei der Melancholie sind so markant, dass keine Energie mehr für die Außenwelt bleibt. Es handelt sich um ein schwarzes Loch in der Psyche, das die libidinöse Energie aufsaugt. Dieses Paper von Freud deutet zwei bedeutende Entwicklungen in der Theorie der Psychoanalyse an, nämlich den Todestrieb und die Ob- jekttheorie, und dies sagt aus, dass die Psyche nichts Homogenes ist sondern aus internalisierten Objekten besteht die Echos, Schatten, Karikaturen oder Übertreibungen wichtiger Menschen aus früher Kindheit und Kleinkindalter des Betroffenen sind. Freud postuliert, dass Selbstvorwürfe vormals Vorwürfe an geliebte Menschen waren, die später durch Internalisierung ins Ego verwandelt worden sind. Er legt hier seine Ansicht dar, dass Melancholie gegen sich selbst gerichteter Sadismus und Hass in Aktion sind. Aufgrund dieses Sadismus' liegt in der Melancholie eine Tendenz zum Suizid.

Das macht es offensichtlich, dass der Sekundärgewinn der Erkrankung darin liegen könnte, die Nahestehenden mit der Krankheit zu quälen. Bemerkenswerterweise befindet sich nach Freud das Objekt, das die Krankheit verursacht hat, oft in der nächsten Umgebung der Patientin/des Patienten.

Es scheint nun, dass das, was zu Zeiten Freuds Melancholie war, heute Depression heißt, was zu einem weiteren Diskurs innerhalb der Theorie der Psychoanalyse einlädt. Das sehr erhellende Paper von Emmy Gut (1985) schlägt vor, sich mit der „Depressionsarbeit" zu befassen, das heißt mit den defensiven Funktionen der Depression und der „normalen Reaktion von Depression“. Man könnte in diesen Texten den Begriff Depression durch Trauer ersetzen und sie würden ihre Gültigkeit nicht verlieren.

Emmy Gut (1985) schreibt, dass depressive Reaktion ein adaptiver Affekt ist, der einer speziellen Abwehrfunktion dient. Die verschiedenen depressiven Reaktionen, das Signal, das sie vermitteln, und die vielen unbewussten kognitiven Anstrengungen, die sie mobilisieren, können wir als „Depressionsarbeit“ bezeichnen. Man kann sagen, dass der Prozess der Depression, wie der jedes anderen Affekts, als ein Versuch der Kommunikation betrachtet werden kann, teils an das Selbst gerichtet mit den Themen des Selbst und unserer Wahrnehmung anderer; teils an andere gerichtet mit unserer Sichtweise von ihnen und von uns selbst.

Küchehoff (2017) betont, dass „das depressive Erleben mit der Angst vor dem Beziehungsverlust“ (S. 25) verbunden ist. Er impliziert, dass Depressionskranke eine „Grundangst vor Trennung“ haben, er schreibt, „die depressive Leere lässt Zeit ,ohne Fülle‘ verrinnen und die ,Erfahrung der Vergangenheit und Vergangenen gewinnt eine Übermacht' gegenüber Gegenwart und Zukunft“ (S. 27).

Böker (2017) nennt als psychodynamische Konstellation des depressiven Grundkonflikts die emotionale Unerträglichkeit einer Beziehungserfahrung von Verlassenwerden und aus Objektbedürftigkeit und Objektenttäuschung, einschließlich der Bemühungen, das Objekt zurückzugewinnen und/oder das zu vermeiden, was die Beziehung gefährden könnte bzw. an eine Enttäuschung am Objekt erinnern könnte; ein „permanenter physiologischer Stress angesichts 
ständiger Bindungsbemühungen, und andrängender Enttäuschungwut" (S. 63).

Man könnte vermuten, dass die Erfahrung des Verlassen-Seins traumatisierend ist und viel psychologischen Stress erzeugen kann, was, unter anderem, in Depression resultieren kann. Aber was bedeutet das im Zusammenhang von generationsübergreifend weitergegebenem Trauma?

Wir lernen durch den Holocaust viel über generationsübergreifend weitergegebene Traumata. Bemerkenswerterweise ist die Psychoanalyse selbst eine Holocaustüberlebende, Wenn wir die Überlegung anstellen, dass unabgeschlossenes oder gescheitertes Trauern ein Grund für Depression ist, können wir verstehen, warum so viele analytische Gedanken sich um verzögertes oder unabgeschlossenes Trauern drehen (Prince 2009).

Überlebende massiver historischer Traumata sind durch das Chronische ihres Zustandes in ihren Fähigkeiten Symbole zu verwenden, frei zu assoziieren, $\mathrm{zu}$ reflektieren und verbalisieren massiv beschädigt (Salberg und Grand 2017, S. 19). Dieses Thema verursacht sogar einen „Generationenwechsel“ in der Psychoanalyse, die Forderung an das analytische Paar, über eine Generation hinauszugehen. Eine andere signifikante Erweiterung betrifft die Reaktion auf markante oder überwältigende Ereignisse, so ist Reis (2005) darum bemüht, das analytische Verständnis der Geschichte einer Patientin/eines Patienten um Kultur und kulturell bedeutsame Ereignisse zu erweitern. Derselben Logik folgt Vaughans (2015) „kulturelle Interjektion“, bei der fremde Anschauungen, Motive etc. Menschen beeinflussen, lange, nachdem ein Ereignis stattgefunden hat.

Der Fall von Frau T. ist lediglich ein Beispiel für das reichhaltige Material, mit dem PsychoanalytikerInnen täglich konfrontiert sind. Dori Laub, einer der Pioniere im Bereich der Forschung zu generationsübergreifend weitergegebenen Traumata und selbst Holocaustüberlebender, beschreibt den Zustand seiner Mutter, die ebenfalls eine Überlebende der NS-Konzentrationslager war, als „depressiv mit zahlreichen Somatisierungen“ (Salberg und Grand 2017, S. 25).

Bion formuliert so treffend zum Thema Traumaarbeit: „Es braucht zwei Köpfe (minds) um die allerverstörendsten Gedanken zu denken“ (Ogden 2008, S. 20), und in der therapeutischen Beziehung sind das PatientIn und TherapeutIn. Allerdings suchen oder erhalten bei weitem nicht alle Überlebenden eines Traumas psychotherapeutische Unterstützung. In diesem Fall hätte Bions Gedanke ein anderes Anwendungsfeld - genau, die Familie. Sein Satz könnte diesfalls so weitergehen: ... den Kopf einer/s Überlebenden und den ihres/seines Kindes, oder vielleicht sogar Enkels. Das ist eine mögliche Erklärung für das Auftreten von unerklärlichen Sorgen, panischer Angst und Albträumen bei den Kindern und Enkelinnen/Enkeln von Überlebenden eines Traumas.
Wie funktioniert generationsübergreifend weitergegebenes Trauma? Eine relationale Sichtweise legt nahe: Kinder beobachten ständig die Gesten und Affekte ihrer Eltern und absorbieren das Bewusste und das Unbewusste ihrer Eltern. In den sich verändernden Stadien von Einstimmung und Nicht-Übereinstimmung passen sich die Kinder an die emotionale Präsenz und Abwesenheit ihrer Betreuer/Eltern an und suchen immer nach Bindung (Salberg und Grand 2017, S. 79).

In einem Artikel (2006) schreibt Harris, dass dort, wo Trauer nicht hat stattfinden können, geisterhafte Phänomene auftreten. Faimberg (2005) untersucht eine Geschichte von Identifikationen, die sie als „mit dem Fernrohr durch die Generationen schauen“ bezeichnet. Wardi (1992) hat das Phänomen der „Gedenkkerzen“ entdeckt und beschrieben, das mit der Namensgebung bei Kindern von ermordeten Verwandten zu tun hat. Salberg und Grand (2017, S. 78) nimmt an, dass es drei Generationen dauern kann, bis verstörende Gefühle und Ereignisse containt sind.

In diesem Zusammenhang vergleiche auch Altes Testament (o.J.), 2 Moses 34.7:

... der Gnade bewahrt an Tausenden von Generationen, der Schuld, Vergehen und Sünde vergibt, aber keineswegs ungestraft lässt, sondern die Schuld der Väter heimsucht an den Kindern und Kindeskindern, an der dritten und vierten Generation.

Aus psychoanalytischer Sicht kann der Holocaust (wie jede historische traumatisierende Gräueltat) als eine externe Realität verstanden werden, die in ihrer extremen Gewalt so unwirklich ist, dass es für einen Menschen, der einer solchen Realität ausgesetzt ist, schwer wird, zu unterscheiden, ob er eine schreckliche Fantasie durchlebt oder eine tatsächliche Realität (Bodenstab 2017, S. 42). Die Wunde, die Leere, der ewige Schmerz, das Gefühl von Leere, das so oft von depressiven Patientinnen/Patienten beschrieben wird, ähnelt „lacunae“, einem von Giorgio Agamben geprägten Begriff, der im Mittelpunkt der Holocausterfahrung steht (Laub in Salberg und Grand 2017, S. 31). Dies liegt daran, dass bei der generationsübergreifenden Weitergabe die Transformation und Übergabe von Informationen unausgesprochen und unbewusst erfolgt.

Viele Psychoanalysen haben sich mit dem historischen Trauma und seinen psychischen Folgen beschäftigt.

Dori Laub kommentiert die Psychodynamik von generationsübergreifend weitergegebenem Trauma folgendermaßen: „Ich, der ich zum Aufbewahrungsort der Gefühle von Entsetzen geworden war, die meine Mutter ablehnen konnte, um weiterhin handlungsfähig (zum Überleben) zu sein“..., er beschreibt „ein von Angst überflutetes Innenleben“ (Salberg und Grand 2017, S. 32). Laub hat den eindringlichen Begriff „leerer Kreis“ geprägt (1998, S. 83), was „die Ab- 
wesenheit von Repräsentation, die Auslöschung von Erinnerung, das Gefühl der Leere, die das Kernvermächtnis eines massiven psychischen Traumas sind“ bedeutet. Das ist eine nützliche Metapher, wenn man mit dem Namenlosen, aber Verstörenden arbeitet.

Was passiert mit einem Kind, das einen Elternteil als abwesend, depressiv, unzulänglich erlebt? André Green (1972) beschreibt dieses Phänomen als das der „toten Mutter“, die für das Kind schrecklich ist - und gleichzeitig ist eine solche Mutter unvermeidlich das Objekt der Identifikation. Diese Ambivalenz gegenüber dem verinnerlichten Objekt und seine Beziehung zum Kind stehen im Mittelpunkt des Freudschen Konzepts der Depression (1917).

$\mathrm{Zu}$ Trauma existiert auch im medizinischen Bereich Forschung, so lassen sich im Feld der Epigenetik unterstützende Argumente für das oben Aufgeführte finden. Das Thema ist für eine Darstellung hier allerdings zu komplex, um im Detail beleuchtet werden zu können. An dieser Stelle sei nur kurz erwähnt, dass einiges dafür spricht, dass stressige, traumatische Erfahrungen in der Kindheit tatsächlich die Art, wie ein Gen sich auswirkt, verändern, so Dietz et al. (2011); Malan-Muller et al. (2014); Skelton et al. (2012); van der Knapp et al. (2014).

Yehuda et al. (2014) vertreten die Ansicht, dass eine mütterliche PTBS Auswirkungen auf die differentielle Symptomatik von PTBS, Dissoziation und Depression hat (in Hopenwasser 2017, S. 64).

Unter Berücksichtigung all dessen können wir mit ziemlich großer Sicherheit feststellen, dass generationsübergreifend weitergegebene Traumata tiefgreifenden Einfluss auf die Entwicklung eines depressiven Geisteszustandes haben. Anders betrachtet: jeder von uns ist Mitglied einer Gruppenmatrix, und diese Gruppe kann auch durch Traumata früherer Generationen beeinträchtigt sein. Lassen Sie uns diesen Aspekt näher betrachten.

\section{Überlegungen auf Großgruppenebene}

Nun, da wir über Depression und ihre Verbindungen mit generationsübergreifend weitergegebenem Trauma auf individueller Ebene nachgedacht haben, soll noch das Problem auf gesellschaftlicher Ebene angesprochen werden. $\mathrm{Zu}$ diesem Thema fordert das Buch des britischen Psychoanalytikers Christopher Bollas (2018) „Bedeutung und Melancholie: Leben im Zeitalter der Verwirrung“ zum Nachdenken heraus und inspiriert. Er analysiert Kultur (Literatur, Politik, Theater) der verschiedenen Jahrhunderte, um die kollektiven Axiome der Gruppenpsychologie von heute zu verstehen.

Wir als Menschheit haben tiefgreifende Ergebnisse in Medizin und Bildung erzielt, aber wir haben es gleichzeitig bei so vielen Gelegenheiten verabsäumt, unsere Aggression zu zähmen, dass wir nicht mehr den Luxus haben, uns ohne Verantwortung zu fühlen für das, was wir einander angetan haben, und einfach weiterzumachen.

Wenn PsychotherapeutInnen heute versuchen, die Botschaft, die das Symptom des Patienten, der Patientin ausmacht, zu verstehen, müssen sie eine transgenerative Anamnese vornehmen.

Es könnte angenommen werden, dass jeder von uns zur traumatisierten Gruppe gehört, die ein Echo von Schuld, Scham oder Verleugnung in sich hat. Wir alle tragen unbenannte und unverdaute Teile von „kulturellen und historischen Introjekten“, die depressive Gemütszustände füttern könnten. Psychologische Diagnostik ist ein Produkt des Zeitgeistes, ebenso wie Depression. Bollas argumentiert, dass „gerade so wie ein einzelner Mensch nicht weiß, dass er eigentlich an einer Depression leidet, eine Gruppeein Dorf, eine Stadtregion, eine Nation oder eine globale Gemeinschaft - chronische psychische Probleme erleiden kann, ohne es zu wissen“. Bollas (2018) betont ein unbewusstes Organisationsprinzip, das für Überlebende eines historischen Traumas $\mathrm{zu}$ einem Lebensthema werden kann. Dies wiederum kann zu automatisch erzeugten Geisteshaltungen oder Verhaltensformen innerhalb einer Gesellschaft führen. Als Beispiel nimmt Bollas Afroamerikaner während der Zeit der Sklaverei (und seitdem), als strukturierte Depression als unbewusstes Prinzip, nach dem jeder leben sollte, von einer Generation an die nächste weitergegeben worden ist, aber ähnliche Prozesse sind auch heute beobachtbar (Prolog xx). Psychologische Axiome bilden die Kultur einer Gesellschaft als Mentalität, die die Gruppe definiert. Ein bestimmtes Axiom kann für Jahrzehnte oder sogar Jahrhunderte als unbewusste Denkweise schlummern, die die Geisteshaltung der Gesellschaft beeinflusst (Prolog $\mathrm{xxi}$.

An dieser Stelle soll noch einmal an die Kleinianische „depressive Position“ als notwendige und fortgeschrittenste Stufe der psychischen Entwicklung erinnert werden. Wenn wir sie auf einer Makroebene auf Länder anwenden, könnte sie Licht auf das Problem des Putin-Vandalismus in der Ukraine, auf Syrien und Afghanistan werfen und die europäischen „Taubheit“ bei der Auseinandersetzung mit diesem Aggressor aufklären. Neben offensichtlichen wirtschaftlichen Überlegungen spielt psychodynamisch gesehen auch dies eine Rolle: ein älteres Kind wird sich nicht freudig mit einem kleineren Kind beschäftigen, das Insekten foltert oder mit dem eigenen Kot spielt. Für das ältere Kind macht das keinen Spaß mehr, es hat die infantile Weltanschauung von Omnipotenz bereits verlassen, weil es schon Erfahrungen mit psychischem Leiden gemacht hat und sich dafür entscheidet, die aggressiven Impulse zurückzuhalten, um anderen Wesen keinen Schmerz zuzufügen. Auf der Ebene einer großen Gruppe (Nation, Land) ist die depressive Position demokratierelevant, es bedarf einer gewissen psychischen Reife, um demokratische Prinzipien zu entwickeln und zu bewahren. 
Der Reifeprozess muss das Denken einschließen, Bollas geht sogar so weit, zu sagen, dass wir „fünfzig Jahre Psychophobie hatten: ein Hass, den eigenen Geist zu betrachten, aus Angst vor dem, was wir dort finden würden. Die Ablehnung von Erkenntnis- und Gesprächstherapien drückt die Angst vor einem Geist (der mit einem Gewissen einhergeht) aus (Vorwort xiii)“. Unerlöste Trauer wird zu Verzweiflung, Desorientierung und Wut. Ein gescheiterter Trauerprozess hat daher die kollektive Melancholie vertieft, die durch die individuelle und kollektive Sinnkrise eingeleitet worden ist. Dies ist passiert, um den Schmerz zu vermeiden, der mit der Erkenntnis der enormen Verluste und des „abstumpfenden“ Teils des Selbst einhergeht, der während zweier Weltkriege erlitten worden ist, so Bollas (2018, S. 26): „die die Seele des Selbst des Westens gelähmt hat“.

\section{Was kann getan werden?}

Erinnern wir uns an Bion, so müssen wir in der Gruppe über Unvorstellbares nachdenken. Psychotherapie, besonders in einer Gruppe, ist nützlich, weil sie Containen, Bezeugen, Aushalten und die Chance auf eine sichere Bindung bietet. Wir müssen die Geschichte, aus der wir hervorgegangen sind, einschließlich ihrer historischen und kulturellen Objekte, verstehen, und dafür brauchen wir Hilfe von kulturellen Formen und Institutionen - „Sozialcontainer“, wie R. Peltz es treffend ausgedrückt hat (2005). Andernfalls ist ein depressiver Geisteszustand oder eine „unproduktive Depression“ (Gut) die einzige Option für uns als Gesellschaft.

Außerhalb eines therapeutischen Kontexts, etwa durch Literatur, Theater, Kunst und Kino, könnten viele der historischen Traumata angesprochen, wenn nicht gar vollständig bearbeitet werden. Auf gesellschaftlicher Ebene existiert die „Zeugenliteratur" um die Vergangenheit $\mathrm{zu}$ zeigen und für die historischen Opfer einzutreten.

\section{Schlussfolgerungen}

Zusammenfassend hat diese Abhandlung hoffentlich aufgezeigt, dass die so weit verbreitete Thematik von Depression ihre Wurzeln auch in der Traumatisierung vergangener Generationen hat. Die Verbindungen zwischen diesen beiden psychischen States sind komplex und nicht auf den ersten Blick ersichtlich. Diese Komplexität lässt sich zum Teil durch das Miteinander-Verschlungen-Sein von Depression und generationsübergreifend weitergegebenem Trauma erklären. Die Autorin hat versucht, einen breiten theoretischen Überblick über das Phänomen zu geben, und es ließe sich der Schluss ziehen, dass die einschlägigen Fachdebatten die Plastizität und Ausweichtendenzen der beiden Phänomene reflektieren.

Depression auf einem persönlichen Level hat oft mit einem Verlust, der Erfahrung, verlassen worden zu sein, nach innen gerichtetem Zorn und Sadismus oder inadäquater Betreuung als Kind zu tun, all das kann ein Trauma verursachen. Wenn ein Elternteil schon traumatisiert ist und keine professionelle Hilfe sucht, ist es möglich, dass sie/er unbewusst das Kind instrumentalisiert. Betrachtet man die schiere Menge an historischem Trauma, der wir in den letzten zwei Jahrhunderten ausgesetzt waren, ist es offensichtlich, dass wir alle unbeweinte Verluste und unbewusste Trauer mit uns tragen. Die Frage, wie weit manche von uns wegen dieser Last des Wissens-und-NichtWissens aus früheren Generationen depressiv werden bedarf weiterer Untersuchungen. Von einem gesellschaftlichen Level aus betrachtet, ist Depression ein Resultat einer Angst des Geistes, eine Abstinenz des Denkens, die die unbewusste generationsübergreifende Weitergabe von psychischen Axiomen befeuert. Und es gibt einen gesellschaftlichen Widerstand dagegen, die eigenen psychischen Idiome zu überdenken. Darüber hinaus ist ein depressiver Geisteszustand die Konsequenz einer Massenamnesie, was zu einer Verkümmerung des Egos führt, ein Prozess, der im Kern der Freudschen „Depression“ liegt (1917).

Die Autorin ist davon überzeugt, dass wir unsere historischen und gesellschaftlichen Wunden aufspüren, benennen und betrauern müssen. Es ist notwendig, die Trauerarbeit zu leisten, um sich von der unproduktiven Depression zu befreien, wie Gut es beschreiben würde. Dieser Prozess mag folgendes umfassen, aber nicht darauf beschränkt sein: klagen, berichten, Zeugnis geben, malen, aufführen - oder auch professionelle Artikel verfassen. Auf die eine oder andere Art haben wir keine Wahl. Die Denkarbeit muss erledigt werden, und die Geschichten müssen erzählt werden! Wir schulden uns dies, denn ohne es gibt es keine Zukunft, die nicht die Vergangenheit wiederholt, und wir wollen nicht mehr dorthin zurück.

Interessenkonflikt M. Lenherr gibt an, dass kein Interessenkonflikt besteht.

Open Access Dieser Artikel wird unter der Creative Commons Namensnennung 4.0 International Lizenz (http:// creativecommons.org/licenses/by/4.0/deed.de) veröffentlicht, welche die Nutzung, Vervielfältigung, Bearbeitung, Verbreitung und Wiedergabe in jeglichem Medium und Format erlaubt, sofern Sie den/die ursprünglichen Autor(en) und die Quelle ordnungsgemäß nennen, einen Link zur Creative Commons Lizenz beifügen und angeben, ob Änderungen vorgenommen wurden.

\section{Literatur}

\section{Verwendete Literatur}

Altes Testament. Bibeltext. Einheitsübersetzung. https://www.die-bibel.de/bibeln/online-bibeln/ einheitsuebersetzung/bibeltext/?no_cache=1. Zugegriffen:5.11.2019 
Böker, H. (2017). Psychodynamische Psychotherapie depressiver Störungen: Theorie und Praxis. Gießen: Psychosozial Verlag.

Bollas, C. (2018). Meaning and Melancholia. Life in the Age of Bewilderment. London: Routledge.

Bundesministerium für Arbeit, Soziales, Gesundheit und Konsumentenschutz. (2019). Gender-Gesundheitsbericht

Dietz, D.M., LaPlan, Q., Watts, E.L., Hodes, G.E., Russo, S.J., Feng, J., Oosting, R.S., Vialou, V., Nestler, E.J. (2011). Paternal transmission of stress-induced pathologies, Biological Psychiatry, 70, 408-414.

Faimberg, H. (2005). The telescoping of generations: listening to the narcissistic links between generations. London: Routledge.

Fonagy, P. (2001). Bindungstheorie und Psychoanalyse. Kap, Bd. 6 (S.90-101). Stuttgart: Klett-Cotta.

Freud, S. (1957). Mourning and melancholia. Standard Edition. Bd. 14 (S. 243-258). London: Hogarth Press. (1917).

Goethe, J.W. von (1949). Faust. Eine Tragödie. In: Goethes Werke. Hamburger Ausgabe in 14 Bänden. Textkritisch durchgesehen und kommentiert von Erich Trunz,Bd.3:Dramatische Dichtungen, Bd. 1. Christian Wegner Verlag, Hamburg 1949, S.7-145. 16. Auflage: C. H. Beck, München 1998, ISBN3-406-31234-9.

Green, A. (1972). The dead mother. In On Private Madness. London: Rebus Press.

Grotstein, J.S. (2007). A Beam of Intense Darkness : Wilfred Bion's Legacy to Psychoanalysis. London: Karnac Books.

Gut, E. (1985). Productive and unproductive depression: interference in the adaptive function of the basic depressed response. British Journal of Psychotherapy. https://doi. org/10.1111/j.1752-0118.1985.tb00929.x

Harris, A. (2006). Ghosts, unhealable wounds and resilience: commentaryon papers bySandraSilverman and Maureen Murphy. Psychoanalytic Dialogues, 16, 543-551.

Hoppenwasser, K. (2017). The Rhythm of Resilience: A Deep Ecology of Entangled Relationality. In Salberg, J., \& Grand, S. Wounds of History: Repair and Resilience in the Trans-Generational Transmission of Trauma. London: Routledge.

van der Knaap, L.J., Riese, H., Hudziak, J.J., Verbiest, M.M., Verhulst, F.C., Oldenhinkel, A.J., van Oort, F.V. (2014). Glucocorticoid receptor gene (NR3C1) methalation following stressful events between birth and adolescence. Transitional Psychiatry, 4, p. e381.

Klein, M. (1940). Mourning and manic depressive states. Int. J. Psychoanal., 21, 125-153.

Korittko, A. \& Pleyer, K. H. (2010) Traumatischer Stress in der Familie. Systemtherapeutische Lösungswege. Göttingen: Vandenhoeck und Ruprecht.

Küchehoff, J. (2017). Depression. Gießen: PsychosozialVerlag.

Laub, D. (1998). The empty circle: children of survivors and the limits of reconstruction. Journal of American Psychoanalytical Association, 46, 507-529.

Lempa, W. (2000). Familientherapie mit Traumapatienten. In F. Lamprecht (Hrsg.), Praxis der Traumatherapie. (S. 145-163). Stuttgart: Pfeiffer bei Klett-Cotta

Mallan-Müller, S., Seedat, S., Hemmings, S.M.J. (2014). Understanding posttraumatic stress disorder: insights from the methylome. Genes, Brain and Behavior, 13, pp. 52-68.

Ogden, T.H. (2008). Bion's four principles of mental functioning. Fort. $D a, 14 B, 11-35$.

Peltz, R. (2005). The manic society. Psychoanalytic Dialogues, $15,321-346$

Prince, R. (2009). Psychoanalysis traumatized: the legacy of the Holocaust. American Journal of Psychoanalysis, 69, 179-194.
Reis, B. (2005). The subject of history/theobject of transference. Studies in Gender and Sexuality, 6, 217-240.

Skelton, K., Ressler, K.J, Norrholm, S.D., Jovanovic, T., BradleyDavino, B. (2012). PTSD and gene variants: new pathways and new thinking. Neuropharmacology, 62, pp.628-637.

Salberg, J., \& Grand, S. (2017). Wounds of History: Repair and Resilience in the Trans-Generational Transmission of Trauma. London: Routledge.

Trepper, T. S. \& Barrett, M. J. (1991) Inzest und Therapie Ein (system)therapeutisches Handbuch. Dortmund: Verlag modernes lernen.

Vaughans, K. (2015). To Unchain Haunting Blood Memories: Intergenerational trauma among African Americans. In: M. O'Loughlin \& M. Charles (Eds), Fragments of Trauma and the Social Production of Suffering: Trauma, History and Memory. (pp. 277-290). Lanham, MD: Rowman \& Littlefield.

Wardi, D. (1992). Memorial Candles. London: Routledge.

Yehuda, R., Daskalakis, N.P., Lehrner, A., Desarnaud, F., Bader, H.N., Makotkine, I., Flory, J.D., Bierer, L.M., Meaney, M.J. (2014). Influences of maternal and paternal PTSD on epigenetic regulation of the glucocorticoid receptor gene in holocaust survivor offspring. American Journal of Psychiatry, 171,pp. 872-880.

\section{Weiterführende Literatur}

Abraham, K. (1911). Notes on the psychoanalytic investigation and treatment of manic-depressive insanity and allied conditions. In: Selected Papers on Psychoanalysis (pp.137-156), 1927. London, Hogarth Press.

Davoine, F. (2007). The characters of madness in the talking cure. Psychoanalytic Dialogues, 17,627-638.

Davoine, F., \& Gaudillière, J. (2004). History Beyond Trauma: Whereof One Cannot Speak, Thereof One Cannot Stay Silent. NewYork, NY: Other Press.

Fenichel, O. (1945).Depression and mania. In: The Psychoanalytic Theory of Neurosis. NewYork, NY: Norton.

Fossion, P., Leys, C., Vandeleur, C., Kempenaers, C., Braun, S., Verbanck, P., Linkowski, P. (2015). Transgenerational transmission of trauma in families of holocaust survivors: The consequences of extreme family functioning on resilience, sense of coherence, anxiety and depression, Journal of Affective Disorders, https://doi.org/10.1016/j.jad.2014. 08.054

Gampel, Y. (1996). The interminable uncanny. In L. Rangell (Hrsg.), Psychoanalysis at the Political Border. R. MosesHrushovski. Madison, WI: International University Press.

Harris, A. (2014). Discussion of Slade's “Imagining Fear.". Psychoanalytic Dialogues, 24, 267-276.

Kandel, E. (2006). In Search of Memory. The Emergence of a New Science of Mind. New York:W.W. Norton \& Company.

Kristeva, J. (1987). Black Sun: Depression and Melancholia. New York, NY: Columbia University Press.

Kultchitskyj, R. (2010). Interview with Oksana Zabuzhko for Ukrainska Pravda. (http://life.pravda.com.ua/society/ 2010/03/22/43789/.Zugegriffen: 1.Sep. 2019

Leuzinger-Bohleber, M., Hatzinger, M., Keller, W. et. al. (2019) Psychoanalytische und kognitiv-behaviorale Langzeitbehandlung chronisch depressiver Patienten bei randomisierter oder präferierter Zuweisung. Ergebnisse der LACStudie. Psyche. Zeitschrift für Psychoanalyse und ihre Anwendungen. LXXIII. Jahrgang, Heft 2, Februar 2019. Hg. Picht, J., Döll-Hentschker, S., King, V. etal.https://doi.org/ $10.21706 /$ ps-73-2-77 
N’Diaye, T. Der verschleierte Völkermord. Die Geschichte des muslimischen Sklavenhandels in Afrika. A. d. Franz. v. Christine und Radouane Belakhdar. Rowohlt, Reinbek.

Salberg, J. (2015). The Texture of Traumatic Attachment: presence and ghostly absence in trans-generational transmissions. Psychoanalytic Quarterly. LXXXIV, Bd. 1 (S.21-46).

Yellow Horse Brave Heart, M. (2019). Historical Trauma. Diversity Foundation. http://new.diversityfoundation.
org/HistoricalTrauma.html. https://doi.org/10.1080/ 02791072.2011.628913.Zugegriffen: 1.Sep 2019.

Zabuzhko, O. (2009). The Museum of Abandoned Secrets. Fact, Kyiv. to English translated by Nina Shevchuk. Murray, Bd. 2012. Seattle, WA:AmazonCrossing.

Hinweis des Verlags Der Verlag bleibt in Hinblick auf geografische Zuordnungen und Gebietsbezeichnungen in veröffentlichten Karten und Institutsadressen neutral. 